\title{
Optimization of Vertical Handoff Decision Algorithm for Wireless Networks
}

\author{
E. Arun, R.S. Moni
}

\section{Elias Arun, R.S. Moni}

Department of Computer Science and Engineering, Department of Electronics and Communication Engineering, Noorul Islam University, Tamil Nadu, India. arunsedly@yahoo.com,r.smoni@yahoo.com

\begin{abstract}
To provide mobile users with seamless access anywhere and anytime there is a strong need forinterworking mechanism between cellular networks and wireless local area networks in the next generation wirelessnetworks. Due to the heterogeneous underlying Quality of Service (QoS) support, the admission traffic in these areashas significant impact on overall resource utilization efficiency and QoS satisfaction when multiple services areconsidered. This paper addresses a Call-Level quality of Service (CLS) vertical handoff algorithm between WLANand cellular networks for seamless ubiquitous access. The CLS involves call blocking/dropping probabilities, meandata transfer rate, and number of handoff per call. Based on the above proposed admission strategy, the admissionregion of a cell or WLAN for the traffic can be derived with the function of new call arrival rate, handoff callarrival rate, and the radius of WLANs. The blocking and dropping probabilities are calculated under the guardchanneladmission strategy. The radius of WLAN is determined by using Simulated Annealing (SA) method to minimizethe cost function. Moreover, handoff traffic should be differentiated from new traffic in terms of call admission. When a Mobile Node (MN) moves from an area, with only cellular coverage to an overlaid WLAN area, theongoing call of the MN should be handed over to the WLAN, and handoff from WLAN to cellular network if theyleave the scope. The results based on a detailed performance evaluation study are presented here to demonstrate theefficacy of the proposed algorithm. Keywords: heterogeneous wireless networks, vertical handoff, seamless mobility, integration handover.
\end{abstract}

\section{Introduction}

The past decade has witnessed the fast evolution and successful deployment of a number of wireless access networks. The two most promising ones are cellular networks and wireless local area network (WLAN). Driven by the service anywhere and anytime concept, it is well accepted that Fourth-Generation $(4 \mathrm{G})$ wireless networks will be heterogeneous, integrating different networks to provide seamless internet access for mobile users with multi mode access capability. One major challenge in cellular/WLAN internet working is how to take advantage of the wide coverage and almost universal roaming support of cellular networks and the high data rates of WLAN. Many issues should be carefully addressed to achieve seamless interworking,such as mobility management, resource allocation, call admission control, security and billing. This article focuses on how to properly admit incoming traffic to the cell or WLAN and when to take handoff decision. The process of switching connections among networks is called handoff. In each network, an admission control policy either accepts the connection request and accordingly allocates the requested bandwidth or blocks the connection request.Higher priority is usually given 
to accept the connection requests from handoff users not the new users. The reason is that from the user's point of view, having a connection abruptly terminated is more annoying than being blocked occasionally on new connection attempts. A service request rejected by its first-choice network can just leave the system or further try to access the other network [1].

There are some related researches on similar problem in two-tier hierarchical cellular networks, in which small size microcells overlay with large macro cells. Many proposed admission strategies [1] [2] are based on user mobility and traffic characteristics. The vertical handoff [3] process involves three stages. The first is the network discovery. In this phase, Mobile Nodes $(\mathrm{MN})$ periodically searches if there are some other different types of wireless networks and take these discovered networks as candidates. The second is the handoff decision phase where MNs compare the state of the current network with candidates, and select one as the handoff target from them according to a certain criterion. The last is the handoff implementation phase where MNs execute the handoff actions and associate with the newly authenticated network. Among these three stages, the handoff decision phase is very important, because it has a direct influence on the network performance and the quality of service of nodes. The objectives of the proposed framework are to maximize network utility through efficient resource allocation, achieve prioritization among different types of connections such as new connections and vertical and horizontal handoff connections, and ensure that the performance of ongoing connections doesn't deteriorate due to accepting too many connections in a service area.

The authors of [4] propose a vertical handoff decision method that simply estimates the service quality for available networks and selects the network with the best quality. In the literature, vertical handoff algorithm is developed in different directions. One of them takes the Received Signal Strength (RSS) and some other factors such as bandwidth, delay and distance into consideration to select the best network through a simple comparison [5] [6]. Another approach utilizes the Artificial Intelligent Techniques such as Neural Network, Fuzzy Logic and machine learning, combining these factors considered to select the best network [7] [8] . The above methods mainly consider the Quality of Service of nodes after handoff, but do not consider the overall system performance such as resource utilization affected by handoff. The servicedifferentiated admission scheme proposed in [9] applies a different admission strategy for voice service, in which the cellular network is the first choice for voice call and no vertical handoff from the cell to the WLAN is executed for ongoing voice calls. To maximize resource utilization, a complex set of admission parameters need to be determined so that the traffic load is properly distributed to the cells and WLANs. This research is based on the resource management and the handoff execution of a node is used to make the resources be optimally utilized [10]. By properly setting the effective bandwidth of services, packet-level QoS such as packet delay and packet loss can be guaranteed, as long as the allocated bandwidth to traffic is no less than the corresponding effective bandwidth requirements.

In the following, the focus is on call-level QoS in terms of call blocking/dropping probabilities, mean data transfer time and number of handoffs per call. Based on the proposed admission strategy, the admission region of a cell or WLAN for the traffic can be derived with the functions of new call arrival rate, handoff call arrival rate and the radius of WLANs. In view of that in a period, the call arrival rates are stable, so it can be represented all the probabilities as functions of the radius of WLAN. The blocking probabilities and the dropping probabilities are calculated under the guard-channel call admission strategy. The radius of WLANs is determined by using Simulated Annealing method to minimize the cost function.

The rest of the paper is organized as follows. Section 2 provides cellular/WLAN system description. Section 3 proposes the optimal admission control for cellular/WLAN. Section 4 proposes the vertical handoff decision algorithm to minimize the call-level QoS. In Section 5 performance of the proposed algorithm is discussed. Finally the conclusion is stated in Section6. 


\section{Cellular/ WLAN System Description}

Consider an integrated cellular/WLAN system where one or more WLANs may be deployed inside each cell of the cellular system as shown in fig.1. There are two specific coverage areas to be considered: the cellular-only coverage area and the dual cellular/WLAN coverage area. In this context, coverage means service availability. A Mobile Node (MN) can be existing at a given time in the coverage area of a cellular alone. But due to mobility it can move into the regions covered by more than one access networks simultaneously within the coverage area of an UMTS BS and an IEEE802.11 AP. Multiple 802.11 WLAN coverage areas are usually contained within an UMTS coverage area. Horizontal and Vertical Handoffs can occur in different coverage areas. In this section it is described a model to formulate a multi-service integrated UMTS/WLAN system.

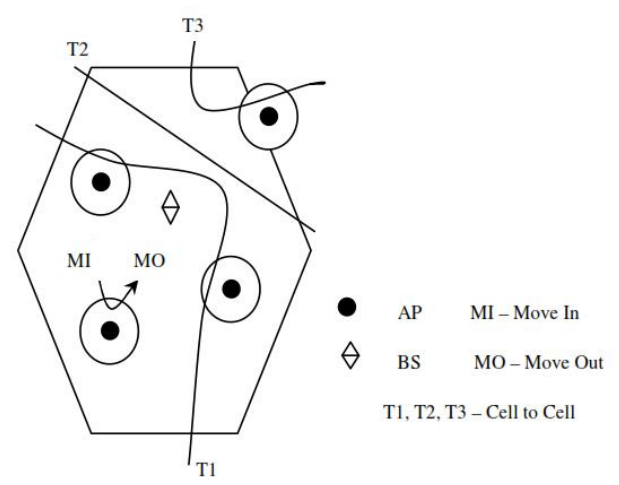

Figure 1: Integrated heterogeneous network

It is assumed that a UMTS network covers $\mathrm{k}$ WLANs and all the WLANs have no overlapping areas and are directly adjacent between two UMTS networks. For simplicity, we only draw a single BS and some APs in fig.1, although there are many other cellular networks besides the cellular network. However this has no influence on the design and analysis of our handoff algorithm.

Let the radii of the UMTS network and WLAN is $r_{u}$ and $r_{w}$ respectively. The number of channels to be $\mathrm{Cu}$ and $\mathrm{Cw}$. WLANs are usually deployed in an indoor environment, where user mobility level is very low and may significantly differ from that of other areas. Hence homogeneous mobility model may not be applicable and it is necessary to differentiate the user mobility characteristics in the double-coverage area from those in the cellular-only area. In the following analysis, a non-uniform model is used to characterize the user mobility within a cell cluster.

Let $t_{\text {res }}^{c o}$ denote the residence time that a user stays within the cellular-only area before moving to neighboring cells with probability $p^{c c}$ or to the overlaying WLAN with probability $p^{c w}$ and $t_{\text {res }}^{d c}$ the user residence time in the double-coverage area. $t_{\text {res }}^{c o}$ and $t_{\text {res }}^{d c}$ are assumed to be exponentially distributed with parameters $\eta^{c o}$ and $\eta^{d c}$, respectively. As shown in [11], for the $\mathrm{MN}$ with mean velocity $\mathrm{V}$ and uniformly distributed movement direction over $[0-2 \pi]$, the average region boundary cross-over rate ç is given by $\eta=V(L / \pi s)$, where $\mathrm{L}$ and $\mathrm{S}$ are the boundary length and area of the region respectively.

To analyze the dwell time, we adapt the third model [12] which is defined as the duration that a node stays in a certain region before it moves out of the boundary of the region, of a node 
in a region. The third model assumes the nodes are uniformly distributed throughout the whole area and each MN moves in any direction with equal probability. The area of the region in the heterogeneous wireless networks that is in double-coverage defined as $S^{d c}$ and boundary length $L^{d c}$. Similarly the area of the region that is covered by a WLAN is defined as $S^{w}$ and boundary length $L^{w}$. The area of the region that is covered by cellular-only area is $S^{c o}$ and boundary length $L^{c o}$. Hence the area $S^{d c}$ equals to the sum of $S^{w}$ and $S^{c o}$. The surface area

$$
S^{c o}=S^{d c}-k S^{w}
$$

where $\mathrm{k}$ is the $k^{\text {th }}$ WLAN.

To model the mobility, it is defined inter-boundary time similar to $[13,14]$, as the time interval between any two consecutive access network boundary crossings by a mobile user. The wider the coverage area or the more stationary users, longer the inter-boundary times. If the inter-boundary time starts at the moment of entering cell $\mathrm{i}$, then it is denoted by $t_{b i}^{c}$. If an interboundary time starts at the moment of entering WLAN $\mathrm{k}$ then it is $t_{b k}^{w}$. It is assumed that $t_{b i}^{c}$ and $t_{b k}^{w}$ are exponentially distributed with means $1 / \eta_{i}^{c}$ and $1 / \eta_{i}^{w}$ respectively. Hence it is noted that the arrival rates of handoff calls and new calls follow poison distribution, the dwell time in a certain region follows exponential distribution and the call duration time follows exponential distribution with mean value as $1 / \eta$. The channel holding time can be defined as the time that a connected mobile user keeps using basic bandwidth resources in each network.

For service $\mathrm{s}$, the channel holding times in cell $\mathrm{i}$ and in WLAN k are obtained $\operatorname{as} \min \left(t_{s}^{R}, t_{b i}^{c}\right)$ and $\min \left(t_{s}^{R}, t_{b i}^{w}\right)$ respectively where $\mathrm{t}_{s}$ is the connection time of a service s. Since $t_{s}^{R}, t_{b i}^{c}$ and $t_{b i}^{w}$ are exponentially distributed, the channel holding times are also exponentially distributed with parameters $\mu_{i s}^{c}=\vartheta_{s}+\eta_{i}^{c}$ and $\mu_{k s}^{w}=\vartheta_{s}+\eta_{k}^{w}$ respectively. With the heterogeneous QoS support of the underlying structure, the incoming traffic in the double-coverage area should be properly admitted to the cell and WLAN. It is assumed that the calls are uniformly distributed in the cellular region, so the call requests in cellular region can be classified as,

(i) New call requests to the WLAN with arrival rate $\lambda_{n}^{w}$ which is equal to $\left(\mathrm{S}^{w} / S^{u}\right) \lambda_{n}$ where $\lambda_{n}$ is the new call arrival rate, and $\mathrm{S}^{u}$ is the surface area of the cell.

(ii) New call request to the cellular-only area with arrival rate $\lambda_{n}^{c o}$ equaling to $\left(S^{c o} / S^{u}\right) \lambda_{n}$.

(iii) New call arrival rate in double-coverage area $\lambda_{n}^{d c}$ which is equal to $\left(S^{d c} / S^{u}\right) \lambda_{n}$.

(iv) The arrival rate of handoff calls between neighboring cell $\lambda_{h}^{c c}$.

(v) The arrival rate of handoff calls from cellular-only area to overlaying WLAN $\lambda_{h}^{c w}$.

(vi) The arrival rate of handoff calls from WLAN to the overlaying cell $\lambda_{h}^{w c}$.

With the above parameters the average dwell time in $\mathrm{k}^{\text {th }}$ WLAN area, $\left(\mathrm{a}_{w}\right)$ is $1 / \eta_{k}^{w}$, where the

$$
\eta_{k}^{w}=V L^{w} / \pi S^{w}
$$

The average dwell time in double-coverage area $\left(a_{d c}\right)$ is $1 / \eta^{d c}$ where

$$
\eta^{d c}=V L^{d c} / \pi S^{d c}
$$

The average dwell time in cellular-only area $\left(a_{c o}\right)$ is $1 / \eta^{c o}$ where

$$
\eta^{c o}=V L^{c o} / \pi S^{c o}
$$

Consider an $\mathrm{MN}$ is in cellular-only area. When $\mathrm{MN}$ is moving out of this region, it may enter into adjacent cell or move into WLAN region. Hence, the average dwell time in cellular-only region before $\mathrm{MN}$ moves into another adjacent cell is $1 / \eta_{h}^{c c}$ where

$$
\eta_{h}^{c c}=V L^{d c} / \pi\left(S^{d c}-k S^{w}\right)
$$


The average dwell time in cellular-only region before MN moves into WLAN area is $1 / \eta_{h}^{c w}$ where

$$
\eta_{h}^{c w}=V L^{w} / \pi\left(S^{d c}-k S^{w}\right)
$$

\section{Optimal Admission Control for Cellular/WLAN Interworking}

With Call Admission Control the heterogeneous network blocks some new call requests in order to reduce interference on the network so that the outage probability decreases. Given the cell bandwidth $C^{c}$ and total offered traffic load, the minimum bandwidth needed to meet the requirements of call blocking and dropping probabilities can be obtained as $x_{n} . N_{n}^{c}$ where $x_{n}$ is the bandwidth requirement of a new call and $N_{n}^{c}$ is the maximum number of new call requests allowed in a cell $\left(N_{n}^{c} \leq C^{c} / x_{n}\right)$. It is noted that in cellular-only region only cellular access is available so in this paper randomized guard channel method [15] is applied to give the new and handoff traffic in this area a priority to access the cell bandwidth over the traffic in the doublecoverage area. Because the call blocking and dropping probabilities are very sensitive to the amount of reserved bandwidth, the guard bandwidth for high priority call traffic is randomized instead of an integer number of guard channels.

Each cellular network has $C^{c}$ channels and each WLAN has $C^{w}$ channels and $C^{c}-N_{n}^{c}$ channels in cellular network are reserved only for handoff calls. Similarly $C^{w}-N_{n}^{w}$ channels in WLAN are reserved only for handoff calls; when the occupied channels are less than $N_{n}^{c}$ in cellular networks, the call admission region of the cell is given in terms of $\left(N_{n}^{c}, G_{h}^{c}, G_{n h}^{c}\right)$ vectors, in which $G_{n h}^{c}\left(\leq N_{n}^{c}\right)$ is a real number to represent a randomized number of guard channels dedicated to new and handoff calls in cellular-only area and $G_{h}^{c}$ is the guard bandwidth reserved only for handoff traffic in this region.

When the occupied channels are equal to or more than $N_{n}^{c}$ only the handoff calls could be allowed; and the same to the WLANs. Within the two-tier overlaying structure, the vertical handoff from the cell to the overlaying WLAN is not necessary but optional to maintain an ongoing call. Hence the handoff traffic load to the WLAN can be controlled by properly adjusting the admission parameters to the WLAN, by using a simple guard channel method. Due to different quality of service support and resource sharing policy in the underlying networks, the configuration of admission regions of the cell and WLAN can have a significant impact on the overall system performance.

Let $P_{b n}^{r e q}, P_{d h}^{r e q}$ and $t_{d}^{r e q}$ are the requirements of new call blocking and handoff call dropping probabilities and mean transfer rate respectively. Then the admission control problem can be formulated as

$$
\max _{N_{n}^{w}} \lambda_{d}
$$

Subject to : $P_{b n}^{w}, P_{b n}^{d c} \leq P_{b n}^{r e q} ; P_{b n}^{c o} \leq P_{b n}^{r e q} ; P_{d h}^{c} \leq P_{b h}^{r e q}, E\left(t_{d}\right) \leq t_{d}^{r e q}$.

Where $P_{b n}^{c o}$ and $P_{b n}^{d c}$ are the blocking probabilities of the cell for new calls in the cellular-only area and double-coverage area respectively, $P_{d h}^{c}$ is the handoff dropping probability of the cell, $P_{b n}^{w}$ is the probability that a new call is blocked by the WLAN and $E\left(t_{d}\right)$ is the mean transfer rate time. Thus the maximization of $\lambda_{d}$ implies a maximization of the total acceptance traffic load and resource utilization.

\subsection{New call blocking and dropping probabilities}

We use a $\mathrm{K}+1$ dimensional Markov Chain to analyze the guard channel admission algorithm. Let $\left(k_{n}^{w}, k_{n c o}^{c}, k_{n d c}^{c}\right)$ denote the state of the new call arrival in a cell cluster, where $k_{n}^{w}, k_{n c o}^{c}, k_{n d c}^{c}$ are the numbers of new calls admitted to the WLAN, to the cell from the cellular-only area and 
to the cell from double-coverage area respectively. First, the number of new calls in the WLAN can be described by a birth-death process with respect to $k_{n}^{w}$. Since both new call duration and user residence time in the double-coverage area are exponentially distributed the channel holding time of new calls in WLANs, $\min \left(t_{s}^{R}, t_{b i}^{c}\right)$ is exponential with mean $1 / \mu_{n}+\eta^{d c}$ where $1 / \mu_{n}$ is the mean new call duration. Then the steady state probability of $\mathrm{k}$ new calls in the WLAN is obtained based on $\mathrm{m} / \mathrm{m} / \mathrm{k} / \mathrm{k}$ loss system, as

$$
\pi_{n}^{w}=\left(\left[\left(\lambda_{n}^{d c}+\lambda_{n}^{c w}\right) /\left(\mu_{n}+\eta^{d c}\right)\right]^{k} / k !\right) /\left(\Sigma_{i=0}^{N_{w}^{w}}\left(\left[\left(\lambda_{n}^{d c}+\lambda_{n}^{c w}\right) /\left(\mu_{n}+\eta^{d c}\right)\right]^{i}\right) / i !\right.
$$

Hence the new call blocking probability in the WLAN is

$$
P_{b n}^{w}=\pi_{n}^{w}\left(N_{n}^{w}\right)
$$

In the following, we derive the state-dependent transition rates, which are given by:

i) $\left(K_{n c o}^{c}, K_{n d c}^{c}\right) \rightarrow\left(K_{n c o}^{c}+1, K_{n d c}^{c}\right)$ and $K_{n c o}^{c}<N_{n}^{c}$.

This happens when there is a new call request in $s^{c o}$ or a handoff call request comes from adjacent cellular networks. Since there is no transition at double-coverage area, it is impossible that a handoff call request comes from one of the WLANs, otherwise $K_{n d c}^{c}$ should be $K_{n d c}^{c}-1$. So the transition rate is

$$
\lambda_{n}^{c o}+\lambda_{h}^{c c}
$$

ii) $\left(K_{n c o}^{c}, K_{n d c}^{c}\right) \rightarrow\left(K_{n c o}^{c}+1, K_{n d c}^{c}\right)$ and $K_{n c o}^{c} \geq N_{n}^{c}$ This will happen when there is a handoff request comes from adjacent cellular network. Because $K_{n c o}^{c} \geq N_{n}^{c}$ it is impossible that a new request call is admitted, and because there is no change of state for the $\mathrm{MN}$ in $K_{n d c}^{c}$ it is also impossible that a handoff call request comes from WLAN. So the transition rate is

$$
\lambda_{h}^{c c}
$$

iii) $\left(K_{n c o}^{c}, K_{n d c}^{c}\right) \rightarrow\left(K_{n c o}^{c}, K_{n d c}^{c}+1\right)$ and $K_{n d c}^{c}<N_{n}^{w}$ this will happen when there is a new call request in the $k^{t h}$ WLAN. Since there is no channel released in cellular network, it is impossible a handoff call request comes from cellular network. So the transition rate is

$$
\lambda_{n}^{w}
$$

iv) $\left(K_{n c o}^{c}, K_{n d c}^{c}\right) \rightarrow\left(K_{n c o}^{c}, K_{n d c}^{c}-1\right)$ and $K_{n c o}^{c}=N_{c}^{c}$ it shows that the channels in WLAN have been used up, the channel released in cellular networks might be driven by three events: call finishes communication; call leaves for neighbor cellular network; call leaves for the k number of used-up-channel WLANs. Hence the transition rate is

$$
K_{n c o}^{c}\left(\mu_{n}+\eta_{h}^{c c}\right)+(K-k) \eta_{n}^{c o}
$$

v) $\left(K_{n c o}^{c}, K_{n d c}^{c}\right) \rightarrow\left(K_{n c o}^{c}, K_{n d c}^{c}-1\right)$ and $K_{n c o}^{c}=C^{c}$ It means that the channels in cellular network have been used up. The channel released in the kth WLAN may be driven by two events: call finishes its communication and call leaves for the cellular network. So the transition rate is

$$
K_{n d c}^{c}\left(\mu_{n}+\eta^{w}\right)
$$

vi) $\left(K_{n c o}^{c}, K_{n d c}^{c}\right) \rightarrow\left(K_{n c o}^{c}, K_{n d c}^{c}-1\right) a n d K_{n c o}^{c}<C^{c}$ This means that the channels in cellular network have not been used up and the channel released in the kth WLAN is only driven by the event that call finishes its communication. It is impossible that a call comes from $S^{w}$ to $S^{c o}$ otherwise $\left(K_{n c o}^{c}\right.$ should be $K_{n c o}^{c}+1$. So the transition rate is 


$$
K_{n d c}^{c} \mu_{n}
$$

As given in equation [12] and [13] the state departure rates vary with the number of existing new calls in WLAN $K_{n}^{w}$ based on which handoff calls from the cell are admitted or blocked by the WLAN. Hence the new calls admitted to the cell from cellular-only region and doublecoverage region has different mean channel holding time. Therefore, the cell can be viewed as a multiservice loss system [16].

A product-form state distribution exists and is insensitive to service time distributions, provided that the resource sharing among services is under coordinate convex policies. This requires that transitions between states come in pairs. For loss systems with trunk reservation like guard channel method, the insensitivity property and product-form solutions are destroyed due to the one-way transitions at some states. A recursive method is proposed in [17] to approximate the state distribution, which is shown to be accurate for a wide range of traffic intensities and when the service rates do not greatly differ from each other.

The blocking probabilities are almost insensitive to service time distributions. Hence, we use the recursive approximation in [17] to obtain the steady- state probability of new calls admitted into the cell $\pi_{n}^{c}$. Thus the blocking probabilities of the cell for new calls in the cellular-only region and double-coverage region are given by

$$
\begin{gathered}
P_{b n c o}^{c}=G_{h}^{c}-\left\lfloor G_{h}^{c}\right\rfloor \pi_{n}^{c}\left\lfloor N_{n c o}^{c}\right\rfloor+\sum_{i=\left\lfloor N_{n c o}\right\rfloor+1}^{N_{n}^{c}} \pi_{n}^{c}(i) \\
P_{b n d c}^{c}=G_{n h}^{c}-\left\lfloor G_{n h}^{c}\right\rfloor \pi_{n}^{c}\left\lfloor N_{n d c}^{c}\right\rfloor+\sum_{i=\left\lfloor N_{n d c}\right\rfloor+1}^{N_{n}^{c}} \pi_{n}^{c}(i)
\end{gathered}
$$

and the dropping probabilities of the cell are given by

$$
D_{n}^{c}=\pi_{n}^{c}\left(N_{n}^{c}\right)
$$

\subsection{Average arrival rates of Handoff calls}

The handoff arrival rates are related to the handoff probabilities. The handoff probability of new calls in the cellular-only area to neighbouring cells is denoted as $H_{n c}^{c c}$ is given by $P^{c c} \varphi\left(-\mu_{n}\right)$ where, $\varphi$ is the moment generating function. Similarly, the handoff probability of new calls in the cellular-only area to the overlaying WLAN is denoted as $H_{n c}^{c w}$ is given by $H_{n c}^{c w}=P^{c w} \varphi\left(-\mu_{n}\right)$ With an exponentially distributed user residence time in the double-coverage area, the handoff probability of new calls from WLAN to the overlaying cell is

$$
H_{n c}^{w c}=\eta^{d c} /\left(\eta^{d c}+\mu_{n}\right)
$$

Hence the handoff traffic from the WLAN to the overlaying cell has a mean arrival rate $\lambda_{h}^{w c}$ is given by

$$
\lambda_{h}^{w c}=H_{n c}^{w c}\left(\lambda_{n}^{d c}+\lambda_{h}^{c w}\right)\left(1-P_{b n}^{w}\right)
$$

The mean arrival rates of handoff traffic between neighbouring cells is

$$
\lambda_{h}^{c c}=H_{n c}^{c c}\left[\lambda_{n}^{c o}\left(1-P_{b n}^{c o}\right)+\left(\lambda_{h}^{w c}+\lambda_{h}^{c c}\right)\left(1-P_{d h}^{c o}\right)+\lambda_{n}^{d c c}\left(1-P_{b n}^{d c}\right) H_{n}^{w c}\right]
$$

And the mean arrival rates of handoff traffic from the cell to the overlaying WLAN is 


$$
\lambda_{h}^{c w}=H_{n c}^{c w}\left[\lambda_{n}^{c o}\left(1-P_{b n}^{c o}\right)+\left(\lambda_{h}^{w c}+\lambda_{h}^{c c}\right)\left(1-P_{d h}^{c o}\right)+\lambda_{n}^{d c c}\left(1-P_{b n}^{d c}\right) H_{n}^{w c}\right]
$$

Thus the new call blocking and dropping probabilities can be obtained recursively from $(9),(18),(20),(21)$ and $(22)$.

\section{Vertical Handoff Decision Making Algorithm}

From the analysis in section 3, it is learnt that if the radius of WLAN is fixed, the drop probability and the block probability will be determined by new call arrival rate and handoff call arrival rate. Usually, the arrival rates in a short period are invariable. Hence the radius of WLAN can be adjusted by regulating the transmission power to optimize these block probabilities and drop probabilities. In order to accomplish an optimization, we formulate a combined cost function

$$
G=\pi_{n}^{c}+\beta_{1} \pi_{n}^{c}+\beta_{2} \lambda_{h}^{w c}+\beta_{3} \lambda_{h}^{c c}+\beta_{4} \lambda_{h}^{c w}
$$

which is determined by the block probabilities and the drop probabilities in cellular networks and one of the WLAN, and is a function of the radius of WLANs. When the radius of WLAN is determined, all the nodes in the WLAN should communicate with WLAN. Even if the channels in WLAN are used up and there are free channels in cellular networks, the call request in WLAN should not be admitted by cellular network would be blocked or dropped. The communication node shall handoff to WLAN if it enters WLAN and handoff to cellular network if it leaves current WLAN. In the equation (23), $\beta_{k}, \mathrm{k}=1,2,3,4$ denotes the weight of dropping probability in WLAN, handoff probability in WLAN to cellular network, handoff probability in cellular-only area to adjacent cell and handoff probability in cellular network to WLAN respectively.

As to the new call originates in $a_{w}$ it is covered by cellular network and couldn't have been admitted by cellular network. According to the above algorithm it loses the opportunity and this will not be happened much often, so the weight of $\beta_{1}$ should be bigger than one. As the MN moving from $a_{c o}$ to $a_{w}$ the handoff is not required because it could have been using the original channel to communicate, but handoff is required if MN moves from $a_{w}$ to $a_{c o}$ otherwise, it will lose the channel of WLAN. So the weight of $\beta_{4}$ should be somewhat greater than $\beta_{2}$ and $\beta_{3}$. Because terminating an on-going call is far more annoying than refusing to admit a new call from user's point of view, $\beta_{4}, \beta_{3}$ and $\beta_{2}$ should be much bigger than $\beta_{1}$. The objective function and its constraints are,

$$
\min G=\pi_{n}^{c}+\beta_{1} \pi_{n}^{c}+\beta_{2} \lambda_{h}^{w c}+\beta_{3} \lambda_{h}^{c c}+\beta_{4} \lambda_{h}^{c w}
$$

such that $R_{\min } \leq R_{w} \leq R_{\max }$, where $R_{\min }$ and $R_{\max }$ are the minimum radius and the maximum radius of the WLAN.

It is very difficult to determine the optimal radius of WLAN by numerical programming methods. Simulated Annealing (SA) is a stochastic computational technique derived from statistical mechanics for finding near globally minimum-cost solutions to large optimization problems. Finding the global minimum value of an objective function with many degrees of freedom subject to conflict ting constraints is an NP-complete problem.

Therefore, the objective function will tend to have many local minima. A procedure for solving optimization problems of the above nature can be implemented by following the Successive Decent Algorithm by Kirkpatric et al [18] which follows the evolution of a solid thermodynamic equilibrium with a decreasing succession of temperature values. The procedure is as follows:

Step 1: Begin minimization. 
Select an initial radius $r_{i} \in$ E randomly for all WLANs;

Select an initial control parameter $\mathrm{T}$ greater than 0 ;

Select parameter change counter $\mathrm{t}=0$;

Repeat;

Set repetition counter $\mathrm{k}=0$;

Repeat.

Step 2: Select a new radius $r_{n}$ in $r_{i}-6, r_{i}+6$ randomly, $r_{n}$ is in $\left[R_{\min }, R_{\max }\right]$;

Compute $\mathrm{S}=G\left(r_{n}\right)-G\left(r_{0}\right)$.

Step 3: If $\mathrm{S}$ less than 0 then $r_{0}:=r_{n}$ goto step 5 .

Step 4: Else if $\operatorname{rand}(0,1)$ less than $\exp (-S / T)$ then $r_{i}:=r_{n}$.

Step $5: \mathrm{k}:=\mathrm{k}+1$.

Until $\mathrm{k}=\mathrm{R}(\mathrm{t})$

$\mathrm{t}:=\mathrm{t}+1$.

Step 6: If $\mathrm{T}$ greater than 30 , then output $r_{0}$ as the optimal radius.

Otherwise $\mathrm{k}:=0$ and;

goto step 2 .

\section{Performance Analysis}

In this section, we evaluate the performance of the proposed vertical handoff decision algorithm in terms of call blocking/ dropping probabilities. Due to the differentiation of new and handoff traffic in different areas, the analysis is very complex. By applying the call admission control algorithm given in section 3, we can obtain the best configuration for admission parameters to maximize the admissible traffic load with the given cell/ WLAN cluster. Fig.2 shows the relationship between the total acceptance traffic load $\lambda_{d}$ and the maximum number of new calls arrived in the WLAN $N_{n}^{w}$ under blocking probabilities $\leq 0.01$, dropping probabilities $\leq 0.001$ and mean data transfer $\leq 4 \mathrm{~s}$.

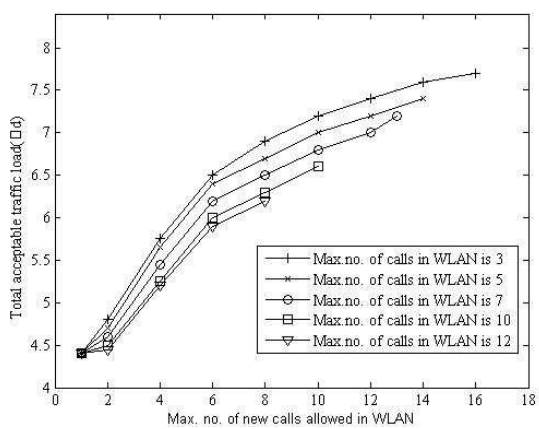

Figure 2: Max. acceptable data traffic load versus max. number of data

It is observed from fig. 2 that the total acceptance traffic load increases with $N_{n}^{w}$ when $N_{n}^{w}$ is relatively small. Fig.3 illustrates the call-level quality of service performance with different $N_{n}^{w}$. It is noted that the simulation results of new call blocking and dropping probabilities are very close to the analytical results. The performance fluctuation of handoff dropping probability is due to the maximum number of calls allowed in the cell and WLAN are both integer variables. From Fig.4 and.5 it is noted that the block probability and drop probability in cellular network 
decreases when the radius of WLAN becomes larger. This is because more and more of new call requests are admitted by WLAN.
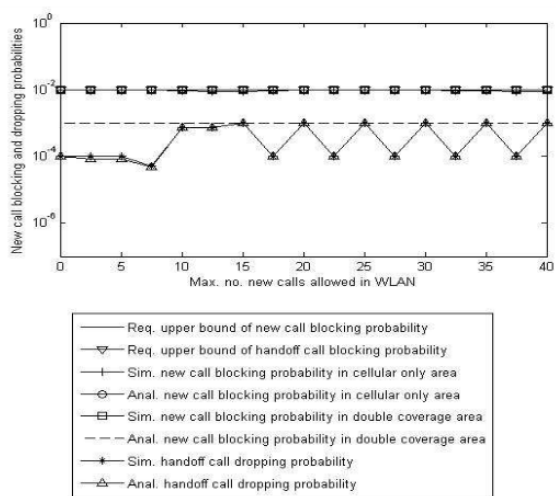

Figure 3: Call level QoS performance with different 5

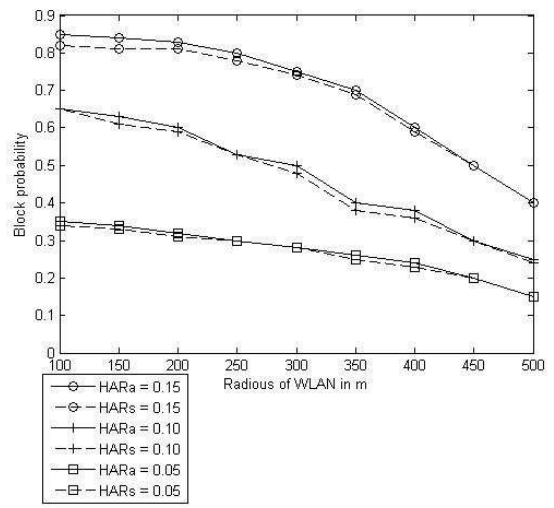

Figure 4: Block probability in cellular networks

Fig. 6 and Fig.7 show that the block probability and drop probability in WLAN becomes larger with the radius of WLAN. The reason here is, when the radius becomes expanding, more and more new calls can be admitted to the WLAN, this will result in increase of block probability and drop probability.

Fig.8 illustrate the optimal radius of WLAN under different new call arrival rate and different handoff call arrival rate. It is observed that when the new call arrival rate is fixed, the radius varies consistent with the handoff call arrival rate. This is because the handoff call requests are only sent to the cellular network, which will increase the drop probability in cellular network, and this leads to maximize the cost function. In order to minimize the cost function, the drop probability must be reduced. This is done by redirecting some of the new call request to WLAN. It is also noted that the handoff call arrival rate is fixed; the radius varies inversely with the new call arrival rate. This is because the number of channels in cellular network is more than that in WLAN. 


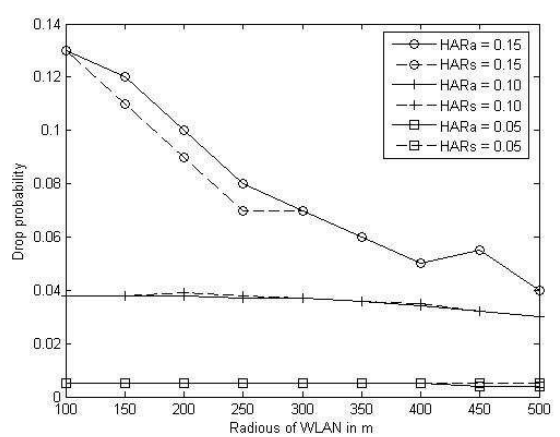

Figure 5: Drop probability in cellular networks

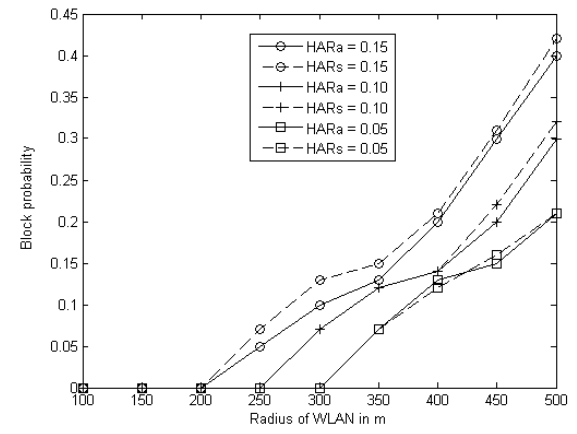

Figure 6: Block probability in WLAN

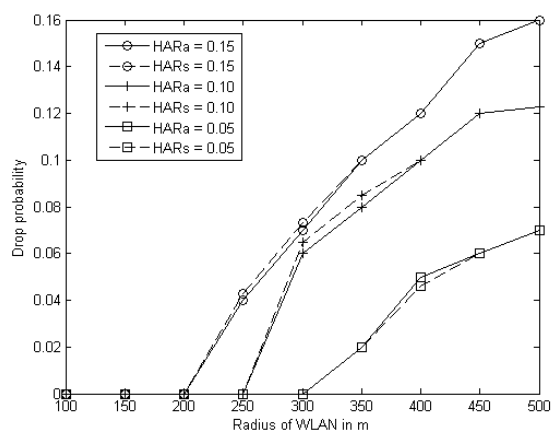

Figure 7: Drop probability in WLAN 


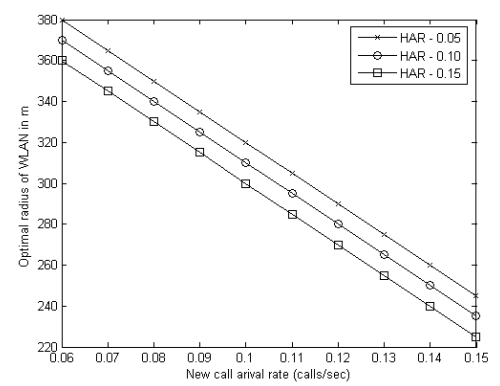

Figure 8: Optimal radius of WLAN

\section{Conclusion}

When connections need to migrate between heterogeneous networks for performance and high availability reasons, then seamless vertical handoff is necessary the first step. In this paper, we tried to highlight the block probability of new calls and drop probability of handoff calls in heterogeneous networks and their computation and the cost function is proposed which is based on the block probabilities and drop probabilities. The optimal radius of WLAN is determined using the simulated annealing method. All the mobile nodes entering the scope of WLAN should handoff from cellular network to WLAN, and the MN leaving the scope should handoff from WLAN to cellular network. Our performance results based on detailed simulations illustrate that the proposed algorithm could achieve good effects.

Acknowledgement.

This work was supported by AICTE, Govt. of India, File No : 8023/BOR/RID/RPS 66/2009-10.

\section{Bibliography}

[1] K.Maheswary and A.Kumar, Performance analysis of microcellization for supporting two mobility classes in cellular wireless networks, IEEE Trans.Veh. Technolog, 49(2):321-333, 2000

[2] T.Klein and S.J.Han,Assignment Strategies for mobile data users in hierarchical overlay networks: Performance of Optimal and adaptive strategies, IEEE Int J. of select. Areas. Commun., 22(5):849-861, June 2004.

[3] J.Ncnair, and F.Zhu, Vertical handoff in 4G multi network environments, IEEE Wireless Communications, 11(3):8-15, 2005

[4] N.Nasser, A.Hasswa and H.Hassanein, Handoff in 4G heterogeneous networks, IEEE communication magazine, 44(10):96-103, 2006

[5] E.Arun, R.S.Moni, A novel decision scheme for vertical handoff in 4Gwireless networks, Global Journal of Computer Science and Technology, 10(5):28-33, 2010

[6] A.H.Zahran, B.Liang, and A.Aaleh,Signal threshold Adaptation for vertical handoff in Heterogeneous wireless networks, vMobile Network Applications, 11:625-640, 2006 
[7] E.Steven, S.Navarro, V.W.S.Wong, and Y.X.Lin, A vertical handoff decision algorithm for Heterogeneous wireless networks, Wireless Communication and networking Conferrence, pp. 3199-3204, 2007

[8] E.Arun, R.S.Moni, Optimization algorithm for a handoff decision in wireless heterogeneous networks, International Journal of Next-Generation Networks (IJNGN), 2(3):99-117, 2010

[9] W.Song and W.Zhuang, QoS provisioning via admission control in cellular/wireless networking, in Proc. 2nd Intl conf. on Broadband Networks (BROADNETS'05), 1:585-593, 2005

[10] P.K.Tang, Y.H.Chew and L.C.O.Michael, Improvement in Grade-of-service in a cooperative overlaying heterogeneous networks, 6th Int conf. on Information Communication and Signal Processing, pp. 1-5, 2007

[11] B.Jabbari, Teletraffic aspects of evolving and next generation wireless communication networks, IEEE Pers. Comm., 3(6):4-9, 1996

[12] Q.A.zeng and D.P.Agerwal, Modeling and efficient handling of handoff in Integrated Wireless Mobile Networking, IEEE Trans. on Veh. Tech., 51(6):1469-1478, 2002

[13] I.Akyildiz and W.wong, A dynamic location management scheme for next generation multityre PCS systems, IEEE Trans. on wireless communications, 1(1):178-189, 2002

[14] D.Hong, and S.S.Rappaport, Traffic model and performance analysis for cellular mobile radio telephone systems with priority and non polarized handoff procedures, IEEE Trans. on Veh. Tech., 35(3):77-92, 1986

[15] N.C.Phuong, S.H.Lee and J.M.Moon, Priority-based call admission control of multi classes in mobile networks, ICACT, pp.1471-1474, 2006.

[16] K.W.Ross, Multiservice loss models for broadband telecommunication networks, Newyork: Springer-verlag, 1995.

[17] P/Tran-Gia and F.Hubner, An analysis of trunk reservation and gradeoff service balancing mechanism in broadband networks, in Proc. TC6 Task group/WG6.4. Int'l. workshop, pp.8397, 1993

[18] Koza.J.R, Genetic programming II automatic discovery of reusable programs, the MIT Press 1994. 